Licença CC BY: Artigo distribuído sob os termos Creative Commons, permite uso e distribuição irrestrita em qualquer meio desde que o autor credite a fonte original.

\section{ANÁLISE BIBLIOMÉTRICA DA PRODUÇÃO CIENTÍFICA DE EMPREENDEDORISMO E TURISMO SUSTENTÁVEL}

\author{
BIBLIOMETRIC ANALYSIS OF SCIENTIFIC PRODUCTION ON \\ ENTREPRENEURSHIP AND SUSTAINABLE TOURISM
}

\section{ANÁLISIS BIBLIOMÉTRICO DE LA PRODUCCIÓN CIENTÍFICA EN EMPRENDIMIENTO Y TURISMO SOSTENIBLE}

PEDRO CHAPAVAL PIMENTEL' ${ }^{1}$

RIVANDA MEIRA TEIXEIRA ${ }^{1}$

'UNIVERSIDADE FEDERAL DO PARANÁ, CURITIBA, PARANÁ, BRASIL

DATA DE SUBMISSÃO: 01/12/2019 - DATA DE ACEITE: 29/04/2020

\begin{abstract}
RESUMO: Sendo o turismo altamente dependente e intimamente ligado a questões ambientais, econômicas e sociais, pesquisadores argumentam que o setor do turismo necessita ser, ao mesmo tempo, sustentável e empreendedor para se manter no longo prazo. Apesar de o empreendedorismo e o turismo sustentável serem campos de pesquisa que vêm recebendo notável atenção acadêmica quando tratados de forma individual, verifica-se a existência de lacuna de estudos que tentem examinar esses dois campos de forma conjunta. Para alcançar este objetivo, foi realizada uma análise bibliométrica em 1.655 artigos e revisões publicadas entre os anos de 1978 e 2019 em periódicos indexados na base de dados Web of Science. O esforço empreendido nesta pesquisa justifica-se pela necessidade de se gerar demandas tanto na esfera acadêmica quanto prática com a análise de ações que estimulem a criação de novos negócios sustentáveis. Os resultados encontrados indicam que o número de publicações segue uma tendência crescente. Amparados na análise de co-citações, é possível identificar que a literatura sobre empreendedorismo e turismo sustentável possui grande parte da sua base teórica fundamentada em autores que dão ênfase à questão do turismo sustentável, deixando o empreendedorismo como aspecto secundário.
\end{abstract}

PALAVRAS-CHAVE: turismo sustentável; empreendedorismo; análise bibliométrica

ABSTRACT: As tourism is highly dependent on, and closely linked to environmental, economic and social issues, researchers argue that the tourism sector needs to be both sustainable and entrepreneurial if it is to sustain itself in the long term. Although entrepreneurship and sustainable tourism are research fields that, individually, have received remarkable academic attention, there have been few studies that attempt to examine these two fields together.

\footnotetext{
PEDRO: Professor substituto do Departamento de Comunicação Social, Universidade Federal do Paraná, Curitiba, Paraná, Brasil. Doutorando em Administração, Universidade Federal do Paraná, Curitiba, Paraná, Brasil. E-mail: professorchapaval@gmail.com. Orcid: 0000-0003-4321-903X.

RIVANDA: Professora no curso de Administração e no Programa de Pós-Graduação em Administração, Universidade Federal do Paraná, Curitiba, Paraná, Brasil. Doutora em Administração, Cranfield University, Cranfield, Bedfordshire, Reino Unido. E-mail: rivandateixeira@gmail.com. Orcid: 0000-0003-3056-6032.
} 
To achieve this goal, a bibliometric analysis was performed of 1,655 articles and reviews published between 1978 and 2019 in indexed journals in the Web of Science database. This research is justified by the need to generate demand in both the academic and practical spheres with the analysis of actions that stimulate the creation of new sustainable business. The results indicate that the number of publications is increasing. Supported by the analysis of co-citations, it is seen that the theoretical base of much of the literature on entrepreneurship and sustainable tourism relies on authors who emphasize the issue of sustainable tourism, leaving entrepreneurship as a secondary aspect.

KEYWORDS: sustainable tourism; entrepreneurship; bibliometric analysis

RESUMEN: Como el turismo es altamente dependiente y está estrechamente relacionado con los problemas ambientales, económicos y sociales, los investigadores sostienen que el sector turístico debe ser sostenible y emprendedor para sostenerse a largo plazo. Aunque el emprendimiento y el turismo sostenible sean campos de investigación que han recibido una atención académica notable cuando tratados individualmente, hay la escassez de estudios que examinen estos dos campos conjuntamente. Para lograr este objetivo, se realizó un análisis bibliométrico en 1.655 artículos y revisiones publicadas entre 1978 y 2019 en revistas indexadas en la base de datos de Web of Science. El esfuerzo realizado en esta investigación se justifica por la necesidad de generar demandas tanto en el ámbito académico como en el práctico con el análisis de acciones que estimulen la creación de nuevos negocios sostenibles. Los resultados indican que el número de publicaciones sigue una tendencia creciente. Apoyado en el análisis de las citas compartidas, es posible identificar que la literatura sobre emprendimiento y turismo sostenible tiene gran parte de su base teórica basada en autores que enfatizan el tema del turismo sostenible, dejando el emprendimiento como un aspecto secundario.

PALABRAS CLAVE: turismo sostenible; emprendimiento; análisis bibliométrico

\section{INTRODUÇÃO}

A indústria do turismo se tornou uma das mais relevantes e de mais rápido crescimento após décadas de desenvolvimento sustentável e diversificação (Porcu et al., 2019). Responsável pela criação de renda, de empregos, de empresas e pelo desenvolvimento de infraestrutura, essa indústria corresponde a uma contribuição indireta de 10,2\% do Produto Interno Bruto (PIB) mundial (Langford \& Weissenberg, 2018 UNWTO, 2018). De forma crescente, somente em 2017 foram mais de 1.340 bilhões de turistas internacionais e um montante de US $\$ 1.447$ trilhões em gastos no setor (The World Bank, 2019).

Devido às rápidas mudanças na economia mundial, a busca, a identificação e a exploração de oportunidades empreendedoras se tornaram cada vez mais importantes para a criação de riqueza (LI, 2008). O crescimento do turismo nas últimas décadas promoveu milhões de novos empregos, aumentou a prosperidade econômica e auxiliou na promoção da cultura local de diversas nações e regiões (Simons-Kaufmann et al., 2012). Contudo, a expansão dessa indústria ocorreu de forma, muitas vezes, descontrolada e trouxe consequências ambientais, sociais e culturais indesejadas (Angelkova et al., 2012). 
A indústria do turismo se tornou um campo fértil para o desenvolvimento de negócios e, consequentemente, para o empreendedorismo (Li, 2008). Ainda que o empreendedorismo como campo teórico seja contestado devido à sua fragmentação, algumas características se destacam; caso da relação entre indivíduos e oportunidades em diferentes contextos (Moroz \& Hindle, 2012; Shane \& Venkataraman, 2000). Logo, a identificação e a exploração de questões relativas ao desenvolvimento sustentável emergem como oportunidades empreendedoras (J. K. Hall et al., 2010).

Com a publicação do Relatório Brundtland (CMMAD, 1991) e os avanços das discussões e na institucionalização de questões relativas ao desenvolvimento sustentável, o empreendedorismo passou a ser reconhecido como conduíte para a criação de negócios sustentáveis (J.K. Hall et al., 2010). Semelhantemente, percebeu-se que o setor do turismo não teria capacidade de se manter no longo prazo se não fosse ao mesmo tempo sustentável e empreendedor (Crnogaj et al., 2014).

A intersecção nas pesquisas sobre empreendedorismo e turismo sustentável pode gerar demandas não apenas na esfera dos negócios, mas também acadêmica. A partir da necessidade de esforços reflexivos sobre as implicações da circulação do conhecimento, da legitimação e da ação para o turismo sustentável e do empreendedorismo (Crnogaj et al., 2014; C. M. Hall, 2019; Işık et al., 2019), busca-se com este trabalho responder a seguinte pergunta de pesquisa: como se caracteriza a produção científica de estudos que tratam do empreendedorismo e do turismo sustentável de forma conjunta?

Dada a escassez de estudos bibliométricos que tratem do empreendedorismo no turismo (Ali et al., 2016; Li, 2008) e a inexistência de estudos revisionais que avaliem o empreendedorismo e o turismo sustentável de forma conjunta, esse estudo pretende trazer contribuições no âmbito acadêmico e gerencial ao analisar ações que estimulem a criação de negócios sustentáveis. Utilizam-se métodos bibliométricos com base em artigos coletados na Web of Science, uma das principais fontes de dados científicos (Mongeon \& Paul-Hus, 2016) para analisar citações, co-citações, coautoria e coocorrência de palavras.

O artigo está dividido em seis seções. Após esta introdução, apresenta-se a revisão de literatura sobre empreendedorismo e turismo sustentável. Em seguida, são analisados estudos revisionais e bibliográficos sobre as temáticas em questão. A quarta seção explica os procedimentos metodológicos adotados neste trabalho, seguida pela apresentação, análise e discussão dos dados encontrados. Por fim, traçam-se as considerações finais, trazendo as limitações e apontando as direções para futuras pesquisas.

\section{EMPREENDEDORISMO}

O estudo do empreendedorismo remete inicialmente a autores como Richard Cantillon e Jean Baptiste Say, que são considerados os pioneiros. Mas é a partir do pensamento econômico da Escola Austríaca que o corpo de conhecimento começa a tomar forma ao avaliar a sua função na criação de novos mercados por meio da 
liderança e inovação, da identificação e exploração de oportunidades lucrativas, e das habilidades, incertezas e tomada de riscos (Landström \& Harirchi, 2018).

Ao focar no fenômeno do empreendedorismo, Schumpeter (1934) avaliou que o crescimento econômico resulta não da acumulação de capital, mas em inovações e novas combinações que criam desequilíbrios no mercado. O estado de desequilíbrio geraria motivações para a busca de oportunidades. Kirzner (1973), por sua vez, entendeu que é fundamental para o indivíduo empreendedor identificar e lidar com diferentes oportunidades, elemento que ele chama de alerta empreendedor. O indivíduo busca desequilíbrios e ineficiências temporais e espaciais resultantes da assimetria de informação no mercado, e deve coordenar recursos de forma efetiva para mitigar ineficiências. Knight (2012) enxerga que oportunidades emergem da incerteza relacionada a mudanças, e que o empreendedor seria um tomador de riscos visando ao lucro.

Com o passar dos anos, o interesse da disciplina ocorreu sob a lente teórica das ciências comportamentais. McClelland (1961) buscou compreender por que algumas sociedades eram mais dinâmicas do que outras e, ao apresentar conexões entre a necessidade de conquista e desenvolvimento econômico, percebeu no empreendedor umimportante vetor para tais fins. Aldrich e Zimmer (1986) apresentaram, sob a perspectiva evolutiva, a necessidade que empreendedores têm de buscar e utilizar laços sociais a fim de atrair recursos e competir no mercado. Stintchcombe (2000) avaliou diferenças entre firmas estabelecidas e firmas em estágio inicial enfatizando o ônus da novidade (liability of newness), que envolve dificuldades no estabelecimento de novas firmas.

Embora a base teórica sobre empreendedorismo tenha sido importada de disciplinas como a economia, a psicologia e a sociologia, e a natureza dos modelos teóricos atuais seja considerada fragmentada, é possível identificar quatro pontos de convergência naquilo que é considerada a essência do empreendedorismo (Işık et al., 2019; Moroz \& Hindle, 2012): (i) a produção científica sobre empreendedorismo avançou junto com as práticas de mercado e vem crescendo por meio de trabalhos empíricos e teóricos (Sarasvathy, 2001; Shane \& Venkataraman, 2000; Venkataraman, 1997); (ii) a relação entre indivíduos e oportunidades é essencial para sua compreensão (Moroz \& Hindle, 2012); as oportunidades lucrativas e empreendedores individuais são elementos sine quae non ao empreendedorismo, campo que envolve o estudo do processo em que indivíduos descobrem, avaliam e exploram fontes de oportunidades lucrativas (Shane \& Venkataraman, 2000; Venkataraman, 1997); (iii) o processo empreendedor envolve a avaliação da criação de valor para partes interessadas em novos modelos de negócios e/ou a otimização de modelos de negócios existentes; e (iv) os aspectos temporal e contextual das ações importam para sua compreensão; as oportunidades não duram para sempre e a receptividade do mercado varia conforme momento e contexto (Moroz \& Hindle, 2012).

Movidos pelo desejo de serem bem-sucedidos e fazer a diferença, empreendedores aproveitam oportunidades oriundas da emergência da indústria turística para transformá-las em bens ou serviços rentáveis; a atividade empreendedora se faz ainda mais relevante (Crnogaj et al., 2014; Işık et al., 2019; Li, 2008). Seja resultado 
de empresas ineficientes, pressões institucionais, falhas em mecanismos de precificação ou distribuição imperfeita de informação, o desenvolvimento de negócios de turismo e de turismo sustentável passou a ser campo frutífero na prática e na teoria (Angelkova et al., 2012; Li, 2008).

\section{TURISMO SUSTENTÁVEL}

A indústria do turismo é composta por negócios que proporcionam bens e serviços diversos para turistas. Isto é, o foco está em pessoas que passam temporadas não superiores a um ano consecutivo em locais diferentes da sua residência para diversos tipos de atividades (Pizam, 2009). Essa indústria compreende atividades que apresentam maiores níveis de crescimento em relação aos demais setores econômicos e requer grande envolvimento de empreendedores devido ao seu alcance local, nacional e mundial (Crnogaj et al., 2014; Işık et al., 2019). Como explicam Simons-Kaufmann (et al., 2012), o sucesso em negócios turísticos vai além do charme do povo local ou estilo de vida tradicional em uma localidade, pois turistas são altamente sensíveis ao valor econômico de suas férias.

Contudo, a diversificação de bens e serviços turísticos passou a sofrer transformações nas demandas; mudanças demográficas, socioculturais, econômicas, tecnológicas e ambientais resultaram em novas oportunidades de negócios para o setor e os impactos ambientais e socioculturais passaram a ser considerados por turistas que se tornaram críticos para essa indústria (Butler, 1980; Simons-Kaufmann et al., 2012).

Como resultado, o conceito de desenvolvimento sustentável passou a ser estudado e aplicado na prática nessa indústria (Crnogaj et al., 2014). A sustentabilidade passou a representar um desafio para as indústrias no mundo todo, pois oportunidades para inovar em diversos setores e negócios receberam uma atenção crescente desde a publicação do Relatório Brundtland (CMMAD, 1991; Niñerola et al., 2019).

A ideia de que o desenvolvimento sustentável é "aquele que atende as necessidades do presente sem comprometer a possibilidade das gerações futuras atenderem as suas próprias necessidades" (CMMAD, 1991, p. 46) gerou transformações nos negócios. A principal preocupação da sustentabilidade, então, tornou-se mitigar impactos humanos que ameaçam a sua própria sobrevivência e a dos ecossistemas aos quais eles são dependentes (Buckley, 2012).

De forma crescente, desde a década de 1980, emergiu a importância de que firmas criassem, planejassem, adaptassem e difundissem inovações que beneficiem aspectos relativos à sustentabilidade (Klewitz \& Hansen, 2014). Desse conceito surgiram negócios sustentáveis (Davies \& Chambers, 2018), econegócios e negócios ambientais (Linnanen, 2002), negócios verdes (Walley et al., 2002) e negócios e empresas sociais (Sassmannshausen \& Volkmann, 1990).

Ainda que a possibilidade de organizações alcançarem objetivos econômicos, sociais e ambientais (triple-bottom line) simultaneamente seja questionada, há evidências de que práticas sustentáveis afetam a percepção e a ação de consumidores (Belz \& Binder, 2017; Simons-Kaufmann et al., 2012; Xu \& Gursoy, 2015). 
Com a popularização do conceito, consumidores se tornaram mais dispostos a adotar estilos de vida e de consumo sustentáveis (He et al., 2018). Organizações inovadoras e sustentáveis passaram a ser vistas como respostas a pressões institucionais para gerar resultados econômicos com responsabilidade social e ambiental, seja em etapas ou em conjunto (Belz \& Binder, 2017).

Ao representar desafios para distintas indústrias, o conceito de sustentabilidade, de acordo com Melissen (2013), pode e deve ser relacionado com atividades ligadas à indústria turística pela sua alta relevância econômica e pelos índices de impacto ambiental e social das suas atividades. O turismo sustentável surge da justaposição entre turismo e sustentabilidade, podendo ser entendida como um tipo de turismo baseado nos princípios do desenvolvimento sustentável que leva em consideração os futuros impactos sociais, ambientais e econômicos e que, simultaneamente, supre as necessidades atuais dos stakeholders (Crnogaj et al., 2014).

Turismo sustentável se refere, portanto, a qualquer atividade relacionada ao turismo que contribua: (1) com a produção e a promoção de recursos naturais e ambientais; (2) com valores culturais e sociais; e (3) com a integridade da comunidade local (Angelkova et al., 2012; Choi \& Sirakaya, 2005). Embora se verifique a existência de uma linha condutora sobre a ideia de turismo sustentável, as mudanças conjunturais e na indústria fazem com que o turismo sustentável seja um conceito carente de definição precisa (Angelkova et al., 2012). Faz-se necessária, portanto, reflexão para compreender as implicações da circulação do conhecimento, e da legitimação e da ação para o turismo sustentável (C. M. Hall, 2019).

\section{ESTUDOS SOBRE TURISMO SUSTENTÁVEL E EMPREENDEDORISMO}

O uso de estudos bibliométricos e revisionais para avaliar a produção, mapear a estrutura, o desenvolvimento e a evolução de determinados campos de estudo é crescente nos últimos anos (Ali et al., 2016). Desse modo, destacam-se, a seguir, pesquisas que tratam de turismo sustentável e empreendedorismo em turismo.

O artigo revisional sobre turismo sustentável mais referenciado na base da Web of Science (Buckley, 2012) explica que, em 2012, a literatura sobre turismo apresentava mais de 150.000 publicações, sendo aproximadamente 5.000 relevantes para o turismo sustentável. Dessas, Buckley (2012) realiza uma revisão de 250 artigos, verificando que respostas e indicadores de impactos sociais e ambientais são temas trabalhados pela indústria do turismo. Entretanto, o autor verifica a inexistência de resultados expressivos em práticas sustentáveis adotadas por empresas turísticas, pois a regulamentação governamental é o principal vetor em prol da sustentabilidade; aspectos econômicos são relevantes, mas respostas em termos de práticas sustentáveis restringem-se à compliance, às manobras políticas, ao marketing e às relações públicas (Buckley, 2012).

O editorial da edição especial do periódico Journal of Cleaner Production (v. 111, Part B, 2016) tem como objetivo compreender se a pesquisa acadêmica sobre sustentabilidade no turismo é valiosa, em quais áreas ela é útil, e aponta perspectivas para o futuro (Budeanu et al., 2016). Avaliando os 25 artigos dessa edição, os autores 
ampliam a percepção de que o turismo pode ser empregado como suporte à promoção da sustentabilidade, pois há uma variedade de frentes para isso, seja em relação às mudanças climáticas, segurança alimentar, ou mesmo instabilidades políticas. Budeanu et al. (2016) defendem a necessidade de um aprendizado entre indústrias com esforços para aumentar a comunicação entre disciplinas científicas e stakeholders.

A fim de avaliar a literatura que trata das relações entre turismo e impacto econômico, Comerio e Strozzi (2019) realizaram um estudo que combina a revisão sistemática de literatura com a análise de redes bibliográficas. Ao avaliar 1.234 artigos, os autores verificaram que o turismo é uma forma de potencializar o crescimento econômico de diversos países, e identificaram três clusters de pesquisa: (1) a exploração da interdependência entre turismo e outros segmentos da economia nacionais e/ou regionais, e da avaliação dos impactos econômicos ligados a grandes eventos; (2) o nexo entre turismo e pobreza, visando identificar ferramentas para reduzir a pobreza e avaliar impactos sociais e ambientais intangíveis do turismo; e (3) validações empíricas do crescimento turístico impulsionado pela economia (Comerio \& Strozzi, 2019).

Yu, Wang e Marcouiller (2019) fazem uma revisão sistemática da literatura sobre turismo que gere benefícios para pessoas em áreas atingidas pela pobreza, o que é denominado como pro-poor tourism (Goodwin, 2008). Destacando que estudos exploram a efetividade e os padrões desse tipo de turismo, os autores alertam para a escassez de revisões quantitativas e realizam uma abordagem bibliométrica. Com base em 164 artigos publicados entre 2002 e 2018, seis temas principais foram identificados (desenvolvimento, gestão de turismo, turismo sustentável, áreas de preservação, pobreza e turismo em favelas) e um framework teórico foi proposto visando incentivar melhores práticas e ampliar possibilidades de pesquisas (Yu et al., 2019).

Niñerola et al. (2019) realizaram uma análise bibliométrica em 4.647 artigos publicados sobre turismo e sustentabilidade entre 1987 e 2018. Os autores identificaram cinco aspectos relevantes nesse "subcampo de pesquisa": (i) a literatura sobre sustentabilidade no turismo está crescendo significativamente; (ii) apesar de seis artigos acumularem mais de 300 citações, ainda há poucos autores produtivos; (iii) das 614 fontes incluídas na revisão, as mais importantes publicaram $46,7 \%$ dos artigos; (iv) os Estados Unidos lideram em número de publicações e citações; e (v) a sustentabilidade vem se tornando uma estratégica para empresas e destinos turísticos (Niñerola et al., 2019).

Dentre os estudos sobre empreendedorismo no turismo, Solvoll, Alsos e Bulanova (2015) mencionam a importância do empreendedorismo para a inovação e criação de valor, mas afirmam que a literatura sobre os temas permanece dispersa. Para preencher essa lacuna, os autores examinaram artigos publicados entre 2000 e 2012 e identificaram duas tendências do empreendedorismo no turismo: (1) uma abordagem convergente, na qual os estudos se amparam no referencial teórico sobre empreendedorismo para analisar o turismo; e (2) uma abordagem divergente, que advoga por um conceito específico de empreendedorismo turístico (Solvoll et al., 2015). 
Fu et al. (2019), ao analisarem pesquisas sobre empreendedorismo em hospitalidade e turismo $(H \& T)$, afirmam que o campo ainda está em processo de evolução. Ao avaliar a literatura em termos micro (indivíduo empreendedor), meso (firmas) e macro (ambiente), os autores sugerem que o empreendedorismo em H\&T é um campo rico na prática, mas ainda carece de desenvolvimento teórico mais profundo, em especial em abordagens críticas como a desenvolvida no artigo (Fu et al., 2019).

A revisão de Işık et al. (2019) estudou relações entre turismo e empreendedorismo. Ao aplicar métodos bibliométricos para avaliar 142 artigos publicados entre 1994 e 2018 , os autores concluíram que as principais temáticas trabalhadas são: (i) pequenas e médias empresas, (ii) tipos de empreendedorismo, e (iii) estudos sobre empreendedorismo na indústria do turismo. Além disso, os autores identificaram um crescimento de publicações a partir de 2010, e que os países mais estudados são África do Sul, China e Austrália.

Se até 2008 os estudos que tratam do empreendedorismo no campo do turismo permaneciam sub-estudados tanto em termos de desenvolvimento teórico quanto empírico (Li, 2008), ao avaliar artigos bibliométricos em turismo sob uma perspectiva crítica, Ali et al. (2016) explicam que esse tipo de pesquisa recebeu atenção crescente entre 2008 e 2015. A ênfase nesse período, contudo, foi dada a revisões sistemáticas e o campo ainda sofre uma escassez de estudos que trabalhem com análises relacionais (Ali et al., 2016). Diante da necessidade de compreender os referidos campos, apresentam-se na seção seguinte as estratégias metodológicas empregadas para analisar a produção científica de estudos sobre empreendedorismo e turismo sustentável de forma conjunta.

\section{METODOLOGIA}

Com o objetivo de analisar a produção acadêmica dos estudos sobre empreendedorismo e turismo sustentável de forma conjunta, optou-se pelo uso da análise bibliométrica. Considerada uma ciência, a bibliometria envolve o estudo quantitativo e estatístico da produção, da publicação, do uso e da disseminação do conhecimento científico oriundo de bases de dados bibliográficas (Gutiérrez-Salcedo et al., 2018).

Por meio de técnicas estatísticas, ela permite avaliar e monitorar o progresso de campos de estudo por meio da classificação de dados como citações e colaborações, afiliações de autores, palavras-chave, temas discutidos, tópicos relevantes e métodos utilizados (Ali et al., 2016). Como resultado, é possível identificar aspectos relevantes a respeito da estrutura do campo, redes sociais e tópicos relevantes para futuras pesquisas com rigor quantitativo, conforme processo apresentado na Tabela 1 (Zupic \& Čater, 2015). 
Tabela 1: Descrição do processo metodológico

\begin{tabular}{l|l|l}
\hline Etapa & Técnica & Descrição \\
\hline 1 & Projeto de pesquisa & $\begin{array}{l}\text { a. Qual é a pergunta de pesquisa? } \\
\text { b. Escolha do(s) método(s) bibliográfico(s) mais adequado(s) (Tabela 2) }\end{array}$ \\
\hline 2 & $\begin{array}{l}\text { Compilação dos dados } \\
\text { bibliométricos }\end{array}$ & $\begin{array}{l}\text { a. Escolha (ou construção) do banco de dados apropriado e dos critérios de } \\
\text { seleção (Tabelas 3 e 4) } \\
\text { b. Filtragem e exportação dos dados bibliográficos (Tabela 5). }\end{array}$ \\
\hline 3 & Análise & $\begin{array}{l}\text { a. Escolha do software bibliométrico (Pacote Bibliometrix do Software R) } \\
\text { b. Seleção das informações para publicação } \\
\text { c. Identificação de grupos e subgrupos por meio do método selecionado }\end{array}$ \\
\hline 5 & Visualização & $\begin{array}{l}\text { a. Escolha do método adequado para visualização } \\
\text { b. Seleção do software para implementar a visualização dos resultados } \\
\text { (Bibliometrix e VOSviewer) }\end{array}$ \\
\hline
\end{tabular}

Fonte: Adaptada de Zupic, I., \& Čater, T. (2015)

A primeira etapa consistiu na definição da seguinte pergunta de pesquisa: Como se caracteriza a produção científica de estudos que tratam de empreendedorismo e turismo sustentável de forma conjunta? No desenvolvimento do projeto de pesquisa foi avaliada a viabilidade de distintos métodos possíveis para análise bibliométricos, tomando como referência o potencial para entregar resultados e unidades de análise, conforme Tabela 2.

Tabela 2: Resumo dos métodos bibliométricos

\begin{tabular}{l|l|l}
\hline Método & Descrição & Unidade de análise \\
\hline Citações & $\begin{array}{l}\text { Estima a influência de documentos, autores ou periódicos por meio } \\
\text { de taxas de citação }\end{array}$ & $\begin{array}{l}\text { Documento, Autor, } \\
\text { Periódico }\end{array}$ \\
\hline Co-citações & $\begin{array}{l}\text { Conecta documentos, autores ou periódicos com base no número } \\
\text { de ocorrências conjuntas em listas de referências em um trabalho }\end{array}$ & $\begin{array}{l}\text { Documento, Autor, } \\
\text { Periódico }\end{array}$ \\
\hline $\begin{array}{l}\text { Acoplamento } \\
\text { bibliográfico }\end{array}$ & $\begin{array}{l}\text { Conecta documentos, autores ou periódicos com base no número } \\
\text { de referências compartilhadas para construção de medidas de } \\
\text { similaridades }\end{array}$ & Documento, Autor Periódico \\
\hline $\begin{array}{l}\text { Coautor } \\
\text { Examina e conecta autores para estabelecer as redes de } \\
\text { colaboração em artigos científicos }\end{array}$ & Autor \\
\hline palavras & $\begin{array}{l}\text { Utiliza palavras para estabelecer relacionamentos quando } \\
\text { aparecem no mesmo documento }\end{array}$ & Palavra e conceito \\
\hline
\end{tabular}

Fonte: Adaptada de Zupic, I., \& Čater, T. (2015)

Os métodos aplicados neste estudo foram: (i) citações, (ii) co-citações, (iii) coautoria, e (iv) co-ocorrência de palavras. A opção desses métodos se justifica para (i) estimar a influência de estudos, autores e periódicos na área de estudo, (ii) verificar a frequência em que unidades de análise são citadas em conjunto, (iii) avaliar as redes sociais de pesquisadores, e (iv) mapear e estabelecer os relacionamentos entre palavras e conceitos. A opção por não aplicar o método de acoplamento bibliográfico se justifica porque seu uso é significativo para analisar publicações no mesmo recorte temporal (Zupic \& Čater, 2015), mas este trabalho avalia publicações entre os anos de 1978 e 2019. 
$\mathrm{Na}$ terceira etapa, foram delimitados palavras-chave, base de dados pesquisada e filtros utilizados (Tabelas 3 e 4). Definiu-se a busca conjunta pelas expressões "Sustainab* Tourism" OR "eco* tourism" OR "social* Tourism" OR "green* Tourism" OR "environm* Tourism" AND "Entrepren*", o que se justifica pelo fato de existirem diversos tipos de negócios com foco sustentável, conforme indicado na seção 2.2. A base de dados escolhida foi a Web of Science e a busca foi limitada a artigos científicos em campos de estudos relacionados aos negócios e à administração (Tabelas 3 e 4). A fim de compreender o campo como um todo, não foi estipulada nenhuma limitação quanto ao recorte temporal.

Tabela 3: Critérios de inclusão e exclusão de artigos

\begin{tabular}{l|l|l}
\hline N. & Critério & Razão para inclusão/exclusão \\
\hline \multicolumn{2}{l}{ Critério de inclusão } \\
\hline 1. & $\begin{array}{l}\text { Publicações que estudam } \\
\text { empreendedorismo ou turismo sustentável }\end{array}$ & $\begin{array}{l}\text { Permitir que se compreende e descreva de forma individual } \\
\text { cada conceito teórico abordado }\end{array}$ \\
\hline 2. & $\begin{array}{l}\text { Publicações que estudem as relações entre } \\
\text { "empreendedorismo" e "turismo sustentável" } \\
\text { de forma conjunta }\end{array}$ & $\begin{array}{l}\text { Permitir compreender as interdependências e relações entre } \\
\text { os conceitos teóricos abordados }\end{array}$ \\
\hline 3. & $\begin{array}{l}\text { Publicações com ênfase na área de } \\
\text { negócios }\end{array}$ & $\begin{array}{l}\text { Permitir compreender as contribuições teóricas feitas por } \\
\text { acadêmicos da área de negócios }\end{array}$ \\
\hline 4. & $\begin{array}{l}\text { Somente artigos e revisões em periódicos } \\
\text { científicos }\end{array}$ & $\begin{array}{l}\text { Permitir argumentos rigorosos e fundamentos teóricos } \\
\text { estabelecidos cientificamente }\end{array}$ \\
\hline Critérios de exclusão & $\begin{array}{l}\text { Publicações em áreas como Medicina, } \\
\text { Psicologia, Enfermagem, Matemática, Física, } \\
\text { Química, Saúde, etc. }\end{array}$ & $\begin{array}{l}\text { Não é o objetivo do artigo explorar pesquisas com foco nesses } \\
\text { temas, pois não proporcionaram informações relevantes ao } \\
\text { propósito definido }\end{array}$ \\
\hline 2. & $\begin{array}{l}\text { Anais de eventos científicos (proceedings } \\
\text { papers) e trabalhos não concluídos (working } \\
\text { papers) }\end{array}$ & $\begin{array}{l}\text { Não é objetivo deste trabalho avaliar artigos que ainda } \\
\text { não foram concluídos ou publicados por periódicos de alto } \\
\text { impacto }\end{array}$ \\
\hline
\end{tabular}

Fonte: Elaborada pelos autores (2019)

A escolha pela base de dados Web of Science ocorreu porque é uma das principais plataformas indexadoras de citações científicas (Mongeon \& Paul-Hus, 2016) e quando foi realizada a busca pelas mesmas palavras-chave na base de dados Scopus, os resultados somaram apenas 126 registros, incluindo artigos, revisões, livros e anais de conferências, inviabilizando a pesquisa quantitativa. 
Tabela 4: Descrição dos termos de busca e resultados em 18 jul. 2019

\begin{tabular}{l|l}
\hline Base de dados & Web of Science \\
\hline & $\begin{array}{l}\text { (TS=("Sustainab* Tourism" OR "eco* Tourism" OR "social* Tourism" OR "green* Tourism" } \\
\text { OR "environm* Tourism" AND "Entrepren*") AND WC=( BUSINESS OR MANAGEMENT OR } \\
\text { ECONOMICS OR PLANNING DEVELOPMENT OR EDUCATION EDUCATIONAL RESEARCH OR } \\
\text { PUBLIC ADMINISTRATION OR SOCIAL SCIENCES INTERDISCIPLINARY OR SOCIAL ISSUES OR } \\
\text { ETHICS OR ENVIRONMENTAL STUDIES OR ENVIRONMENTAL SCIENCES OR GREEN SUSTAINABLE } \\
\text { SCIENCE TECHNOLOGY OR SOCIOLOGY OR ENGINEERING MULTIDISCIPLINARY OR SOCIAL } \\
\text { WORK OR ENGINEERING ELECTRICAL ELECTRONIC OR BUSINESS FINANCE OR COMPUTER } \\
\text { SCIENCE INFORMATION SYSTEMS OR ENGINEERING ENVIRONMENTAL OR OPERATIONS RESEARCH } \\
\text { MANAGEMENT SCIENCE OR MULTIDISCIPLINARY SCIENCES OR HUMANITIES MULTIDISCIPLINARY } \\
\text { OR INTERNATIONAL RELATIONS )) }\end{array}$ \\
\hline Filtros & Article OR ReVIeW \\
\hline Artigos & 1.592 \\
\hline Revisões & 63 \\
\hline Total & 1.655 \\
\hline Amostra analisada & 1.606 \\
\hline
\end{tabular}

Fonte: Elaborada pelos autores (2019)

A partir da linha de busca aplicada na base Web of Science (Tabela 4), foi gerado um total de 1.655 registros (data base: 18 jul. 2019). Destes, 49 artigos foram excluídos da análise porque estavam duplicados ou classificados como anais de eventos científicos (Proceedings Paper), resultando em uma amostra de 1.543 artigos e 63 revisões. Em seguida foram coletadas as seguintes informações: título do artigo, autor(es), instituições às quais o(s) autor(es) estava(m) filiado(s) na data de publicação, país da instituição, ano de publicação, palavras-chave, título do periódico, referências utilizadas.

A terceira etapa consistiu na definição dos softwares que foram utilizados para a análise; o software bibliométrico VOSviewer (van Eck \& Waltman, 2010) e o pacote Bibliometrix (versão 2.2.1.), ferramenta do software livre R (Aria \& Cuccurullo, 2017). A quarta etapa consistiu na visualização e na avaliação de informações que a própria plataforma Web of Science disponibiliza, como autores, instituições de ensino e pesquisa, áreas de pesquisa que mais produziram conhecimento, e língua em que os artigos foram escritos. Em seguida, foram realizados o tratamento dos dados e a exportação (Bibliometrix e VOSviewer) para a análise dos dados e construção de mapas bibliométricos, que permitiram visualizações gráficas. A seção seguinte traz os principais resultados e análises dos dados.

\section{RESULTADOS}

De forma geral, a produção científica sobre empreendedorismo e turismo sustentável de forma conjunta apresenta uma taxa de crescimento anual de 18,34\%. O campo em questão apresenta uma evolução quantitativa que pode ser classificada em quatro estágios (1978-1993; 1994-2003; 2004-2014; 2015-2019), conforme mostra a Figura 1. 
Figura 1: Evolução da produção científica anual de artigos entre 1978 e 2019

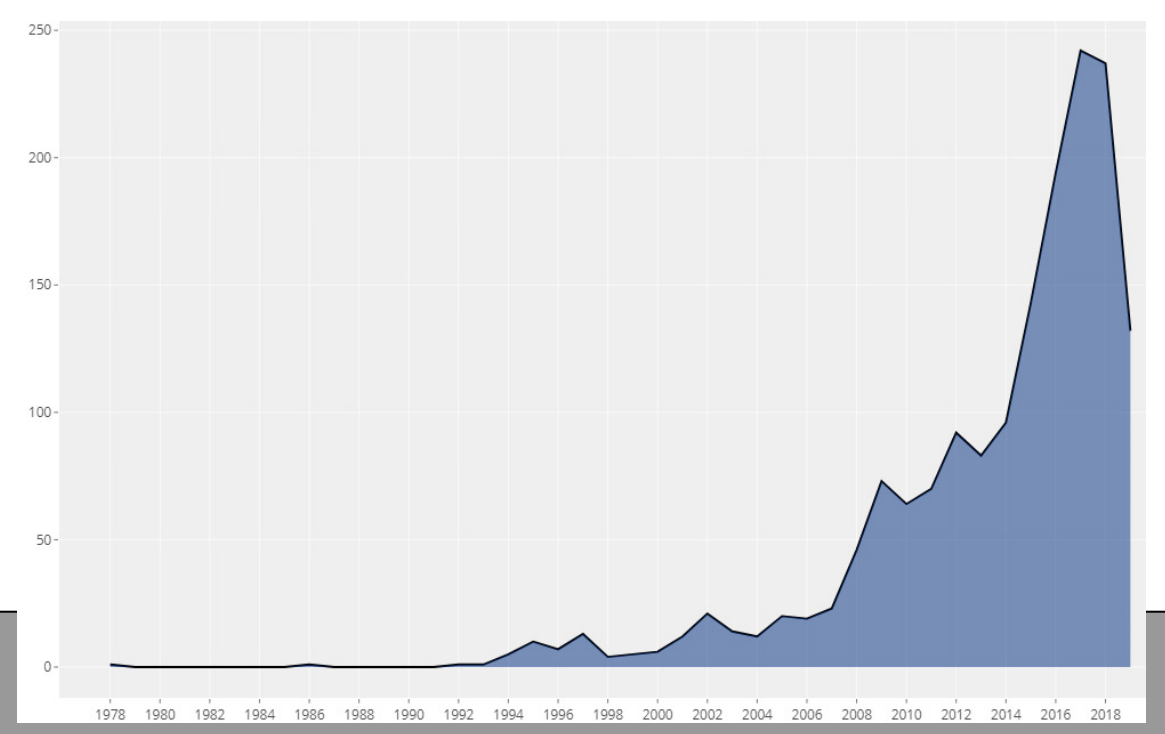

Fonte: Adaptada pelos autores do pacote Bibliometrix (2019)

O primeiro estágio contém artigos publicados entre 1978 e 1993, com publicações esparsas, não mais de uma por ano. Identificaram-se, também, anos em que não houve nenhuma publicação sobre o tema, indicando um campo de pesquisa ainda em estágio embrionário. Diferente de estudos anteriores (Buckley, 2012; Niñerola et al., 2019; Yu et al., 2019), foi possível encontrar publicações anteriores a 1987; a primeira publicada em 1978 trata de aspectos relativos ao turismo social e ao envelhecimento (Rottmann, 1978).

No segundo estágio, entre 1994 e 2003, as publicações ocorreram com maior frequência, numa média de dez artigos por ano. É possível inferir que, a partir da publicação do Relatório Brundtland (CMMAD, 1991), o interesse pelo tema tem aumentado, proporcionando novas lentes de reflexões para os campos do empreendedorismo e do turismo sustentável. Entretanto, somente após dez anos da publicação do Relatório é que os artigos ultrapassaram treze por ano, indicando que a categoria ainda estava nascente, tendo pouco mais de um artigo publicado por mês.

O terceiro estágio ocorreu entre 2004 e 2014, com um aumento gradativo na quantia de artigos científicos publicados, alcançando uma média anual de 54 publicações. A grande mudança na quantia de publicações ocorreu em 2015, quando foram publicados 143 artigos; caracterizando o quarto estágio. É possível inferir que esse aumento foi resultado da publicação do volume especial do Journal of Cleaner Production naquele ano. Conforme mencionado, a edição visava avaliar o engajamento do setor do turismo nos desafios relacionados à sustentabilidade (Budeanu et al., 2016). Desde então foram publicados em média 190 artigos por ano, alcançando 242 publicações em 2017.

No que diz respeito às principais áreas de pesquisa que estudam empreendedorismo e turismo sustentável, há diversidade e interdisciplinaridade. De acordo com a classificação da Web of Science, a área que mais produz é a de Social 
Sciences (757), seguida por Environmental Sciences (692), Science Technology (634), Business Economics (424), e Sociology (136). Logo, ainda que autores como Budeanu et al. (2016) defendam a necessidade de distintas disciplinas estudarem o turismo sustentável, isso já vem sendo realizado. Por outro lado, é possível que problemas conceituais decorram justamente dessa interdisciplinaridade (Angelkova et al., 2012).

Conforme Zupic e Čater (2015), dentro do universo e do método selecionado, é possível realizar a avaliação das fontes mais citadas e que indicam a sua influência no campo. Em outras palavras, se determinado estudo, país, centro de pesquisas ou periódico são muito citados, podem ser considerados importantes para a ciência e para aquele campo de pesquisa (Zupic \& Čater, 2015). Assim, a maioria dos artigos produzidos está escrito em língua inglesa $(94,86 \%)$, sendo o restante $(5,14 \%)$ escrito em espanhol (52 artigos), russo (10), croata (5), português (4), francês (3), malaio (3), entre outros com 1 ou 2 publicações cada; resultados que refletem os principais países produtores de pesquisas na área de empreendedorismo e turismo sustentável (Tabela 5).

Tabela 5: Países com mais artigos publicados e referenciados sobre empreendedorismo e turismo sustentável

\begin{tabular}{l|l|l|l|l}
\hline Ranking & País & Artigos Produzidos & País & Artigos Referenciados \\
\hline 1 & Estados Unidos & 331 & Reino Unido & 4.995 \\
\hline 2 & Austrália & 290 & Austrália & 4.907 \\
\hline 3 & China & 273 & Estados Unidos & 3.699 \\
\hline 4 & Espanha & 242 & Canadá & 2.125 \\
\hline 5 & Reino Unido & 242 & Espanha & 2.125 \\
\hline 6 & Itália & 137 & Suécia & 1.415 \\
\hline 7 & Taiwan & 113 & China & 1.026 \\
\hline 8 & Turquia & 97 & Nova Zelândia & 1.006 \\
\hline 9 & Canadá & 96 & Taiwan & 733 \\
\hline 10 & Romênia & 90 & Noruega & 651 \\
\hline
\end{tabular}

Fonte: Adaptada pelos autores de Web of Science (2019)

Embora a China seja um dos três maiores produtores de pesquisas sobre os temas (Tabela 5) e um dos que mais tem estudos aplicados (Işık et al., 2019), ela não é o país que possui mais artigos citados, ficando apenas na sétima posição. Curiosamente, a produção científica do Reino Unido é mais referenciada do que a dos Estados Unidos, o maior produtor em quantidade de trabalhos. Vale mencionar o papel da Espanha como um dos cinco países que mais produz e mais é referenciado e, simultaneamente, o papel da língua hispânica como a segunda na produção de conhecimento.

Quanto à análise de coautoria, que avalia as redes sociais existentes na produção do conhecimento cientííco, a Figura 2 corrobora com os dados da Tabela 5 , demonstrando que os nós mais fortes se dão nos seguintes países: Estados Unidos, China, Austrália, Inglaterra (Reino Unido), Espanha, Canadá e Nova Zelândia. A coautoria pode ser vista como uma medida de colaboração entre diferentes instituições (Zupic \& Čater, 2015). 
Figura 2: Coautoria entre países de trabalhos sobre empreendedorismo e turismo sustentável

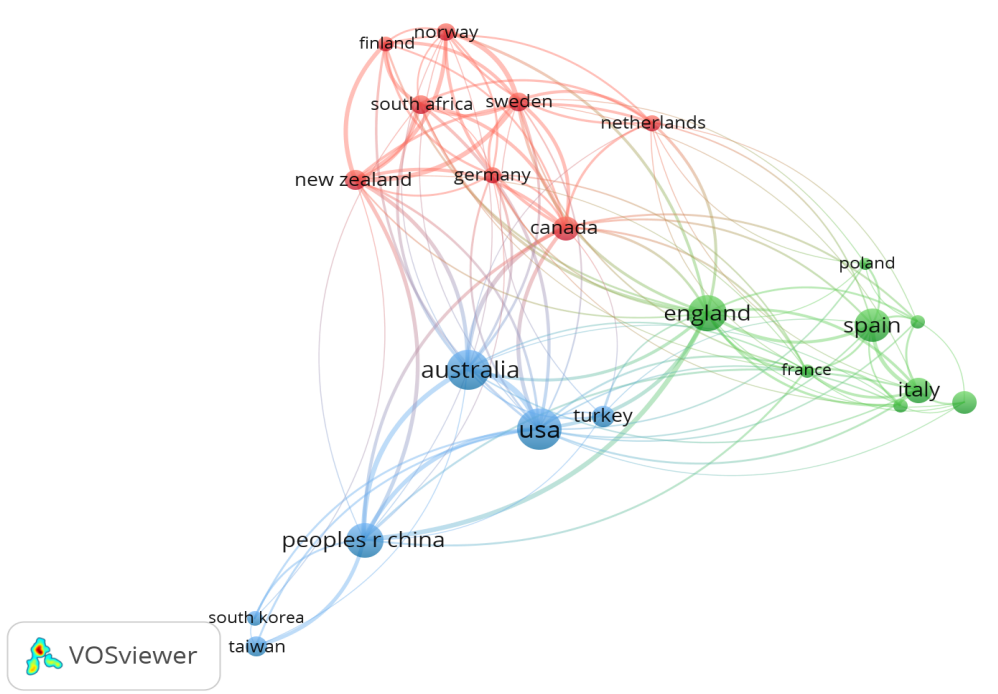

Fonte: Elaborada pelos autores com o software VOSviewer (2019)

É possível verificar a existência de três clusters com países que tiveram ao menos 20 documentos publicados e analisados. Dentre os 110 países, 28 se enquadraram neste requisito e constam na Figura 2. O cluster azul é liderado pelos Estados Unidos, com forte associação com Austrália, China, Turquia, Coréia do Sul e Taiwan. O segundo cluster com associações mais fortes (verde) tem a liderança da Inglaterra associada a Espanha, Itália, França, Grécia, Portugal, Polônia e Romênia. O terceiro cluster (vermelho) tem o Canadá como nó mais forte, seguido de Nova Zelândia, Suécia, África do Sul, Norvega, Finlândia, Países Baixos e Alemanha. Percebe-se, deste modo, a ausência de representantes relevantes das Américas Central e do Sul.

Adicionalmente, os principais centros de pesquisa em empreendedorismo e turismo sustentável estão localizados na Austrália (Griffith University e University of Queensland), Reino Unido (University of Surrey), Espanha (Universidad de Málaga) e Hong Kong (The Hong Kong Polytechnic University). Esses resultados se diferem de Niñerola, Sánchez-Rebull e Hernández-Lara (2019), que trazem como principais produtores e com artigos mais referenciados os Estados Unidos, o Reino Unido, a Austrália, o Canadá e a China. Embora todos os países estejam presentes nesta análise, a diferença de amostragem é um elemento que pode ter ocasionado tal diferença.

Ao que tange os periódicos que mais publicam sobre empreendedorismo e turismo sustentável, estão o Journal of Sustainable Tourism (339 artigos), o Sustainability (148), O Tourism Management (141), o Annals of Tourism Research (90) e o Journal of Cleaner Production (49). Esses dados corroboram com as três principais fontes apresentadas na lista de Niñerola, Sánchez-Rebull e Hernández-Lara (2019), também presentes no estudo de Işik (et al., 2019).

Enquanto Niñerola, Sánchez-Rebull e Hernández-Lara (2019) encontraram seis artigos sobre empreendedorismo e turismo sustentável com mais de 300 citações, este estudo verificou apenas três (Choi \& Sirakaya, 2006; Gössling, 2002; Saarinen, 
2006), conforme Tabela 6. A seleção toma como base o Global Citation Score (GCS), ou seja, o número de citações a um determinado artigo oriundas de todas as fontes possíveis de identificar, conforme reportado no Web of Science na data de análise dos dados. Semelhantemente, uma possível explicação para tais diferenças é o uso de bases de dados, palavras-chaves e filtros diferentes nas buscas entre este artigo e os autores em questão.

Tabela 6: Artigos mais citados que abordam os temas empreendedorismo e turismo sustentável

\begin{tabular}{|c|c|c|c|c|c|}
\hline Rank. & Título & Author & Periódico & Ano & GCS \\
\hline 1 & $\begin{array}{l}\text { Global Environmental Consequences of } \\
\text { Tourism }\end{array}$ & Gossling & $\begin{array}{l}\text { Global Environmental } \\
\text { Change }\end{array}$ & 2002 & 352 \\
\hline 2 & $\begin{array}{l}\text { Sustainability Indicators for Managing } \\
\text { Community Tourism }\end{array}$ & $\begin{array}{l}\text { Choi e } \\
\text { Sirakaya }\end{array}$ & Tourism Management & 2006 & 336 \\
\hline 3 & $\begin{array}{l}\text { Traditions of Sustainability in Tourism } \\
\text { Studies }\end{array}$ & Saarinen & Annals of Tourism Research & 2006 & 307 \\
\hline 4 & $\begin{array}{l}\text { Food, Place and Authenticity: Local Food } \\
\text { and The Sustainable Tourism Experience }\end{array}$ & Sims & $\begin{array}{l}\text { Journal of Sustainable } \\
\text { Tourism }\end{array}$ & 2009 & 291 \\
\hline 5 & $\begin{array}{l}\text { Sustainable Tourism as An Adaptive } \\
\text { Paradigm }\end{array}$ & Hunter & Annals of Tourism Research & 1997 & 287 \\
\hline 6 & Sustainable Tourism: Research and Reality & Buckley & Annals of Tourism Research & 2012 & 254 \\
\hline 7 & Mindful Visitors - Heritage and Tourism & Moscardo & Annals of Tourism Research & 1996 & 253 \\
\hline 8 & $\begin{array}{l}\text { Governance, The State and Sustainable } \\
\text { Tourism: A Political Economy Approach }\end{array}$ & Bramwell & $\begin{array}{l}\text { Journal of Sustainable } \\
\text { Tourism }\end{array}$ & 2011 & 242 \\
\hline 9 & Reconceptualizing Tourism & Farrell et al. & Annals of Tourism Research & 2004 & 239 \\
\hline 10 & $\begin{array}{l}\text { Environmental Management of a } \\
\text { Tourist Destination - A Factor of Tourism } \\
\text { Competitiveness }\end{array}$ & Mihalic & Tourism Management & 2000 & 237 \\
\hline
\end{tabular}

Fonte: Elaborada pelos autores (2019)

Os artigos mais citados cujos temas envolvem turismo sustentável e empreendedorismo tratam de aspectos conceituais relacionados ao turismo e à sustentabilidade (Buckley, 2012; Farrell \& Twining-Ward, 2004; Hunter, 1997; Saarinen, 2006). Os demais tratam de questões gerenciais mais amplas, como é o caso de estratégias (Sims, 2009), indicadores (Choi \& Sirakaya, 2006), governança (Bramwell, 2011 ) e competitividade (Mihalič, 2000). O empreendedorismo, portanto, é tangenciado de forma secundária nesses artigos, um possível reflexo dos debates relacionados à existência do empreendedorismo como um campo ou subcampo de pesquisa (Landström \& Harirchi, 2018; Shane \& Venkataraman, 2000; Young \& Tilley, 2006).

Para além de verificar os artigos mais citados em termos de Global Citation Score, é possível identificar a frequência com que dois ou mais autores foram mais citados juntos em um mesmo artigo (co-citação). Essa medida tem como premissa o fato de que quanto mais determinados itens são citados em conjunto, mais provável é que o conteúdo entre eles seja relacionado (Zupic \& Čater, 2015). Assim, os 20 autores que obtiveram ao menos 150 citações cada (Figura 3) podem ser considerados como mais relevantes para estudos em empreendedorismo e turismo sustentável. 
Figura 3: Mapa de co-citação de autores

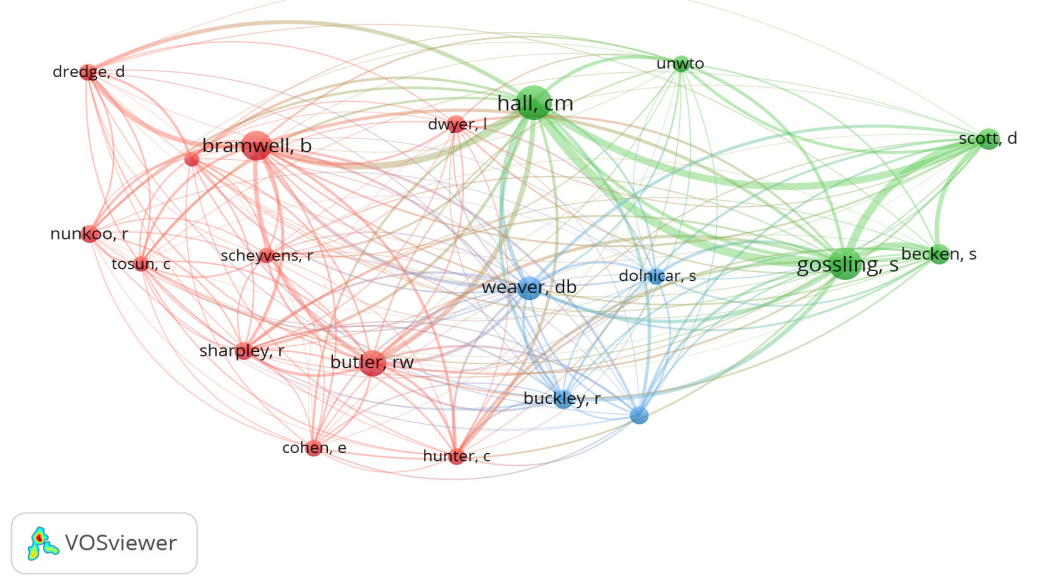

Fonte: Elaborada pelos autores com o software VOSviewer (2019)

De um universo de 38.921 autores citados, no cluster verde os principais são Hall (citado 676 vezes) e Gossling (607), autores de artigos como "Constructing sustainable tourism development: The 2030 agenda and the managerial ecology of sustainable tourism" (C. M. Hall, 2019) e "Global environmental consequences of tourism" (Gössling, 2002). Já no cluster vermelho, os autores mais citados são Bramwell (504) e Butler (407), autores de artigos como "Interpretation and sustainable tourism: The potential and pitfalls" (Bramwell \& Lane, 1993) e "The Concept of A Tourist Area Cycle of Evolution: Implications for Management of Resources" (Butler, 1980). E o cluster azul tem como principais autores Weaver (353) e Buckley (246), autores de "Can sustainable tourism survive climate change?" (Weaver, 2011) e "Sustainable tourism: Research and reality" (Buckley, 2012).

Amparados na análise de co-citações, é possível identificar que a literatura sobre empreendedorismo e turismo sustentável possui grande parte da base teórica fundamentada em autores cujos artigos dão ênfase ao turismo sustentável, deixando o empreendedorismo como aspecto secundário. Embora os dados utilizados para a criação da Figura 3 não se restrinjam aos artigos mencionados, em termos quantitativos a literatura apresenta uma base sólida sobre turismo sustentável, diferente do que foi afirmado por Angelkova et al. (2012).

A Figura 4 apresenta o mapa de co-ocorrência de palavras-chave, que permite estabelecer relações entre as palavras-chave utilizadas e construir uma estrutura conceitual sobre o campo em análise. O resultado desta rede apresenta um mapa semântico que possibilita identificar a estrutura cognitiva do campo (Zupic \& Čater, 2015). É possível verificar no mapa formado 20 palavras que tiveram ao menos 50 menções em um universo de 2.200 . 
Figura 4: Mapa de análise de co-ocorrência de palavras

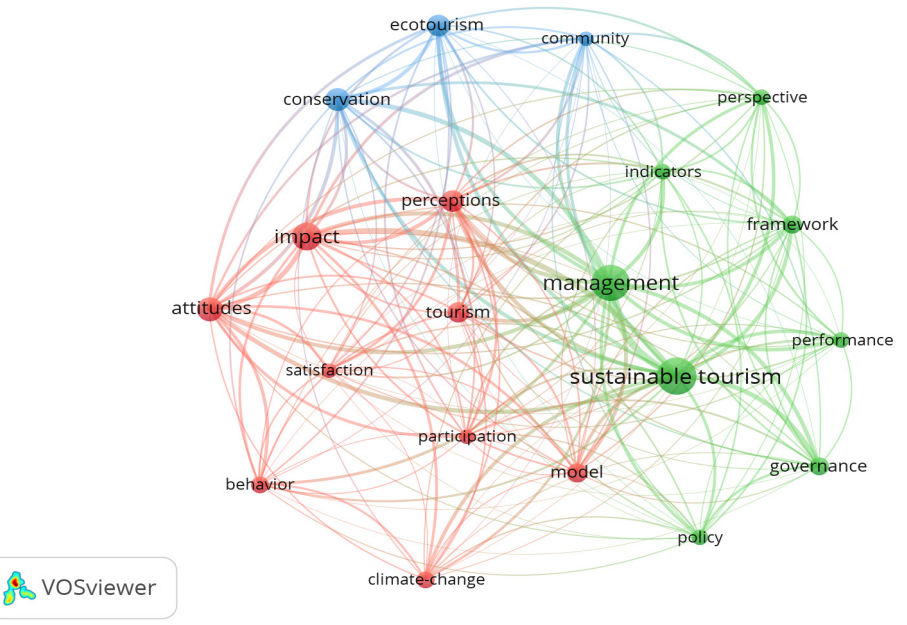

Fonte: Elaborada pelos autores com o software VOSviewer (2019)

A Figura 4 apresenta os três agrupamentos de palavras mais pertinentes aos estudos em empreendedorismo e turismo sustentável de forma conjunta. $\bigcirc$ primeiro deles - verde - tem como principais palavras "sustainable tourism" e "management", seguidas por "governance" (72), "framework" (71), "policy" (58), "performance" (55), "perspective" (52) e "indicators" (52). É possível inferir que os estudos que mais utilizam essas palavras têm como foco empresas em si, o que condiz com as palavras-chave utilizadas para a busca no banco de dados da Web of Science. Compreender aspectos relacionados à performance da firma, dos indicadores, dos modelos e da governança é tema relevante e que demonstra mais força quando o assunto é negócio de turismo sustentável.

O segundo cluster com mais força nas ligações entre os nós é o vermelho e tem como palavras "impact" (152), "attitudes" (118), "perceptions" (94), "tourism" (91), "model" (83), "behaviour" (65), "climate change" (64), "participation" (51), "satisfaction" (50). É possível inferir que a atenção dos artigos neste cluster está na percepção do impacto que determinadas atividades e atitudes relacionadas ao turismo têm na mudança climática. Já o terceiro cluster - azul - apresenta como expressões "conservation" (104), "ecoturism" (93) e "community" (51), e pode ser entendida como estudos que avaliam o ecoturismo como possível prática em prol da preservação do meio ambiente.

\section{CONSIDERAÇÕES FINAIS}

Buscou-se por meio de um estudo bibliométrico compreender a produção acadêmica sobre empreendedorismo e turismo sustentável de forma conjunta. A partir da base Web of Science, foi realizada a análise em 1.655 artigos e revisões publicadas entre os anos de 1978 e 2019. Foi possível verificar que a literatura sobre os temas apresenta maturidade sobre turismo sustentável, com elevado número de artigos, impulsionados pela publicação do relatório Brundtland (CMMAD, 1991) e pela edição especial de um 
dos principais periódicos da área de sustentabilidade, o Journal of Cleaner Production, em 2015. A literatura sobre empreendedorismo e turismo sustentável, quando avaliada em conjunto, apresenta uma base consistente no que diz respeito à quantidade de publicações científicas. Adicionalmente, as pesquisas que envolvem empreendedorismo e turismo sustentável vêm recebendo mais atenção cientíica, pois com base nos dados apresentados verificou-se uma taxa de crescimento anual acima de $15 \%$.

Válido ressaltar que a maioria dos artigos produzidos sobre os temas em conjunto está publicada em língua inglesa, reflexo dos principais países produtores de pesquisas e dos países que mais recebem citação. Deve-se destacar que os estudos sobre empreendedorismo e turismo sustentável em conjunto apresentam características de diversidade e interdisciplinaridade, envolvendo áreas de conhecimento relacionadas às ciências sociais e tecnológicas. Além disso, a análise das palavras-chave mais utilizadas demonstra que o empreendedorismo aparece como tema secundário, e a ênfase dos artigos recai sobre questões gerenciais (management) de negócios em turismo sustentável.

Ao avaliar elementos como os artigos e autores mais citados, os países e os centros de pesquisa que mais publicam, as palavras-chave mais utilizadas e os principais periódicos científicos, faz-se uma contribuição aos pesquisadores em estágio inicial de seus estudos e que se interessem pelas temáticas aqui tratadas ao: (1) apresentar trabalhos seminais para o desenvolvimento de pesquisas; (2) descrever a base conceitual, teórica e metodológica; (3) apontar campos que carecem explicações; e (4) desvelar caminhos promissores para avanço da ciência. Em outras palavras, desse modo é possível, com os dados apresentados, fornecer direções para futuras pesquisas.

Destaca-se a necessidade de pesquisas com ênfase no empreendedorismo para a sustentabilidade na indústria do turismo. Pesquisadores no campo do empreendedorismo, portanto, podem desenvolver estudos que promovam maior compreensão, desenvolvimento e ampliação de aspectos e ferramentas para a promoção e avaliação da sustentabilidade nesses negócios. Avaliar questões ambientais relacionadas a mudanças climáticas, polvição e segurança ambiental, e aspectos sociais aplicados à promoção desenvolvimento econômico e de políticas públicas políticas torna-se necessidade na agenda de pesquisas.

Ainda como sugestões para futuras pesquisas, recomenda-se o estudo de pesquisas que utilizem outras temáticas relacionadas às desta pesquisa, como é o caso das relações do turismo com empreendedorismo ambiental, empreendedorismo sustentável, ecoempreendedorismo e outros. Como limitações, o método empregado aqui não possui a capacidade de identificar redes de conexões entre acadêmicos que façam parte dos mesmos grupos de pesquisa e que publiquem juntos (Üsdiken \& Pasadeos, 1995).

Além disso, por ser uma análise quantitativa, não é possível identificar como cada artigo citado foi tratado e elaborado, se recebeu críticas ou houve maior refinamento nas análises propostas (Zupic \& Čater, 2015). Também não foi possível traçar o caminho das publicações, como elaborado por Comerio e Strozzi (2019), devido à opção metodológica escolhida para este estudo. Nesse sentido, também se recomenda a 
realização de revisões sistemáticas da literatura que possibilitem ampliar e aprofundar as análises aqui realizadas.

Para fins práticos e gerenciais, dada a importância do empreendedorismo para a indústria do turismo (Işık et al., 2019; Solvoll et al., 2015), a necessidade de integração de valores ambientais às atividades empreendedoras (Silveira et al., 2018) e, em especial, na indústria em questão (Buckley, 2012; He et al., 2018), é possível que, a partir dos diferentes caminhos apontados nesta pesquisa, empreendedores encontrem maneiras para a adoção de práticas de turismo sustentável em suas organizações e processos. Logo, a partir dos resultados encontrados neste artigo, espera-se fornecer insights e desvelar possibilidades para a aplicação da sustentabilidade em atividades turísticas para que negócios empreendedores alcancem resultados nas dimensões econômica, social e ambiental.

\section{REFERÊNCIAS}

Aldrich, H., \& Zimmer, C. (1986). Entrepeneurship through Social Networks. In D. L. Sexton \& R. W. Smilor (Eds.), The Art and Science of Entrepeneurship (pp. 3-24). Ballinger. https://doi.org/10.2139/ ssrn. 1497761

Ali, M., Rahimi, R., Okumus, F., \& Liu, J. (2016). Bibliometric studies in tourism. Annals of Tourism Research, 61, 180-198. https://doi.org/10.1016/j.annals.2016.10.006

Angelkova, T., Koteski, C., Jakovlev, Z., \& Mitrevska, E. (2012). Sustainability and Competitiveness of Tourism. Procedia - Social and Behavioral Sciences, 44, 221-227. https://doi.org/10.1016/j. sbspro.2012.05.023

Aria, M., \& Cuccurullo, C. (2017). Bibliometrix: An R-tool for comprehensive science mapping analysis. Journal of Informetrics, 11 (4), 959-975. https://doi.org/10.1016/j.joi.2017.08.007

Belz, F. M., \& Binder, J. K. (2017). Sustainable Entrepreneurship: A Convergent Process Model. Business Strategy and the Environment, 26(1), 1-17. https://doi.org/10.1002/bse.1887

Bramwell, B. (2011). Governance, the state and sustainable tourism: A political economy approach. Journal of Sustainable Tourism, 19(4-5), 459-477. https://doi.org/10.1080/09669582.201 1.576765

Bramwell, B., \& Lane, B. (1993). Interpretation and sustainable tourism: The potential and the pitfalls. Journal of Sustainable Tourism, 1 (2), 71-80. https://doi.org/10.1080/09669589309450706

Buckley, R. (2012). Sustainable tourism: Research and reality. Annals of Tourism Research, 39(2), 528546. https://doi.org/10.1016/j.annals.2012.02.003

Budeanu, A., Miller, G., Moscardo, G., \& Ooi, C. S. (2016). Sustainable tourism, progress, challenges and opportunities: An introduction. Journal of Cleaner Production, 111 (Part B), 285-294. https://doi. org/10.1016/j.jclepro.2015.10.027

Butler, R. W. (1980). The Concept of A Tourist Area Cycle of Evolution: Implications for Management of Resources. Canadian Geographer, XXIV(1), 5-12. https://doi.org/10.1111/j.1541-0064.1980. tb00970.x 
Tur., Visão e Ação, v22, n3, p552-574, Set./Dez. 2020 - Balneário Camboriú, Santa Catarina, Brasil

Choi, H. C., \& Sirakaya, E. (2005). Measuring Residents 'Attitude toward Sustainable Tourism: Development of Sustainable. https://doi.org/10.1177/0047287505274651

Choi, H. C., \& Sirakaya, E. (2006). Sustainability indicators for managing community tourism. Tourism Management, 27(6), 1274-1289. https://doi.org/10.1016/j.tourman.2005.05.018

CMMAD. (1991). Comissão Mundial sobre o Meio Ambiente e Desenvolvimento. Nosso futuro comum. Rio de Janeiro: FGV.

Comerio, N., \& Strozzi, F. (2019). Tourism and its economic impact: A literature review using bibliometric tools. Tourism Economics, 25(1), 109-131. https://doi.org/10.1177/1354816618793762

Crnogaj, K., Rebernik, M., Hojnik, B. B., \& Gomezelj, D. O. (2014). Building a model of researching the sustainable entrepreneurship in the tourism sector. Kybernetes, 43(3/4), 377-393. https://doi. org/10.1108/K-07-2013-0155

Davies, I. A., \& Chambers, L. (2018). Integrating hybridity and business model theory in sustainable entrepreneurship. Journal of Cleaner Production, 177, 378-386. https://doi.org/10.1016/j. jclepro.2017.12.196

Farrell, B. H., \& Twining-Ward, L. (2004). Reconceptualizing tourism. Annals of Tourism Research, 31 (2), 274-295. https://doi.org/10.1016/j.annals.2003.12.002

Fu, H., Okumus, F., Wu, K., \& Köseoglu, M. A. (2019). The entrepreneurship research in hospitality and tourism. International Journal of Hospitality Management, 78(October 2018), 1-12. https://doi. org/10.1016/j.ijhm.2018.10.005

Goodwin, H. (2008). Pro-poor tourism: A response. Third World Quarterly. https://doi. org/10.1080/01436590802215287

Gössling, S. (2002). Global environmental consequences of tourism. Global Environmental Change, 12, 283-302.

Gutiérrez-Salcedo, M., Martínez, M. Á., Moral-Munoz, J. A., Herrera-Viedma, E., \& Cobo, M. J. (2018). Some bibliometric procedures for analyzing and evaluating research fields. Applied Intelligence. https://doi.org/10.1007/s10489-017-1105-y

Hall, C. M. (2019). Constructing sustainable tourism development: The 2030 agenda and the managerial ecology of sustainable tourism. Journal of Sustainable Tourism, 27(7), 1044-1060. https://doi.org/ 10.1080/09669582.2018.1560456

Hall, J. K., Daneke, G. A., \& Lenox, M. J. (2010). Sustainable development and entrepreneurship: Past contributions and future directions. Journal of Business Venturing, 25(5), 439-448. https://doi. org/10.1016/j.jbusvent.2010.01.002

He, P., He, Y., \& Xu, F. (2018). Evolutionary analysis of sustainable tourism. Annals of Tourism Research, 69(July 2017), 76-89. https://doi.org/10.1016/j.annals.2018.02.002

Hunter, C. (1997). Sustainable tourism as an adaptive paradigm. Annals of Tourism Research, 24(4), 850-867. https://doi.org/10.1016/s0160-7383(97)00036-4

Işık, C., Küçükaltanb, E. G., Çelebic, S. K., Çalkınd, Ö., Ensere, İ., \& Çelikf, A. (2019). Tourism and Entrepreneurship: A Literature Review. Journal of Ekonomi, 01, 1-27. 
Kirzner, I. M. (1973). Competiton \& Entrepreneurship. The University of Chicago Press.

Klewitz, J., \& Hansen, E. G. (2014). Sustainability-oriented innovation of SMEs: A systematic review. Journal of Cleaner Production, 65, 57-75. https://doi.org/10.1016/j.jclepro.2013.07.017

Knight, F. (2012). From risk, uncertainty, and profit. In The Economic Nature of the Firm: A Reader, Third Edition. https://doi.org/10.1017/CBO9780511817410.005

Landström, H., \& Harirchi, G. (2018). The social structure of entrepreneurship as a scientific field. Research Policy, 47(3), 650-662. https://doi.org/10.1016/j.respol.2018.01.013

Langford, G., \& Weissenberg, A. (2018). Deloitte: 2018 Travel and Hospitality Industry Outlook.

Li, L. (2008). A review of entrepreneurship research published in the hospitality and tourism management journals. Tourism Management, 29, 1013-1022. https://doi.org/10.1016/j.tourman.2008.01.003

Linnanen, L. (2002). An Insider's Experiences with Environmental Entrepreneurship. Greener Management International, 38, 71-80. https://doi.org/10.9774/gleaf.3062.2002.su.00008

McClelland, D. C. (1961). The Achieving Society. Macmillan Publishing Co., Inc.

Mihalič, T. (2000). Environmental management of a tourist destination: A factor of tourism competitiveness. Tourism Management, 21 (1), 65-78. https://doi.org/10.1016/S0261-5177(99)00096-5

Mongeon, P., \& Paul-Hus, A. (2016). The journal coverage of Web of Science and Scopus: a comparative analysis. Scientometrics, 106(1), 213-228. https://doi.org/10.1007/s $11192-015-1765-5$

Moroz, P. W., \& Hindle, K. (2012). Entrepreneurship as a Process: Toward Harmonizing Multiple Perspectives. Entrepreneurship: Theory and Practice, 36(4), 781-818. https://doi.org/10.1111/ j.1540-6520.2011.00452.x

Niñerola, A., Sánchez-Rebull, M. V., \& Hernández-Lara, A. B. (2019). Tourism research on sustainability: A bibliometric analysis. Sustainability (Switzerland), 11 (5), 1-17. https://doi.org/10.3390/sul1051377

Pizam, A. (2009). What is the hospitality industry and how does it differ from the tourism and travel industries? International Journal of Hospitality Management, 28(2), 183-184. https://doi. org/10.1016/j.jijhm.2009.01.001

Porcu, L., del Barrio-García, S., Alcántara-Pilar, J. M., \& Crespo-Almendros, E. (2019). Analyzing the influence of firm-wide integrated marketing communication on market performance in the hospitality industry. International Journal of Hospitality Management, 80 (January), 13-24. https:// doi.org/10.1016/j.ijhm.2019.01.008

Rottmann, G. (1978). Is recreation for elderly new kind of social turism. Caritas, 79(2), 85-87.

Saarinen, J. (2006). Traditions of sustainability in tourism studies. Annals of Tourism Research, 33(4), $1121-$ 1140. https://doi.org/10.1016/j.annals.2006.06.007

Sarasvathy, S. D. (2001). Causation and Effectuation: Toward a Theoretical Shift from Economic Inevitability to The Academy of Management Review. In Academy of Management Review (Vol. 26, Issue 2, pp. 243-263). https://doi.org/10.5465/AMR.2001.4378020 
Tur., Visão e Ação, v22, n3, p552-574, Set./Dez. 2020 - Balneário Camboriú, Santa Catarina, Brasil

Sassmannshausen, S. P., \& Volkmann, C. (1990). A Bibliometric Based Review on Social Entrepreneurship and its Establishment as a Field of Research. In Schumpeter School of Business and Economics (Vol. 35, Issue 5). Schumpeter School of Business and Economics. https://doi.org/10.1007/BF02821 405

Schumpeter, J. (1934). The Theory of Economic Development. In The Economy as a Whole.

Shane, S., \& Venkataraman, S. (2000). The promise of entrepreneurship as a field of research. Academy of Management Review, 25(1), 217-227.

Silveira, A., Backes, D. A. P., \& Kobayashi, A. R. K. (2018). Sustainable entrepreneurship: the scientific production of the 21 st century. Revista de Negócios, 22 (3), 22-37. https://doi.org/10.7867/19804431.2017v22n3p22-37

Simons-Kaufmann, C., Kaufmann, F., Sloan, P., \& Legrand, W. (2012). Introduction: scarcity of natural resources or "Cockaigne"? In W. Legrand, C. Simons-Kaufmann, \& P. Sloan (Eds.), Sustainable hospitality and tourism as motors for development: case studies from developing regions of the world (pp. 1-23). Routledge.

Sims, R. (2009). Food, place and authenticity: Local food and the sustainable tourism experience. Journal of Sustainable Tourism, 17(3), 321-336. https://doi.org/10.1080/09669580802359293

Solvoll, S., Alsos, G. A., \& Bulanova, O. (2015). Tourism Entrepreneurship - Review and Future Directions. Scandinavian Journal of Hospitality and Tourism, 15, 120-137. https://doi.org/10.1080/15022250. 2015.1065592

Stinchcombe, A. L. (2000). Economics Meets Sociology in Strategic Management. In J. A. C. Baum \& F. Dobbin (Eds.), Economics Meets Sociology in Strategic Management (Vol. 17, pp. 229-259). Emerald Group Publishing Limited. https://doi.org/10.2307/3094810

The World Bank. (2019). International tourism data. In World Bank Open Data. http://data.worldbank. org/indicator/ST.INT.XPND.CD

UNWTO. (2018). Tourism Highlights, 2018 Edition. https://doi.org/10.18111/9789284419876

Üsdiken, B., \& Pasadeos, Y. (1995). Organizational Analysis in North America and Europe: A Comparison of Co-citation Networks. Organization Studies, 16(3), 503-526. https://doi. org/10.1177/017084069501600306

van Eck, N. J., \& Waltman, L. (2010). Software survey: VOSviewer, a computer program for bibliometric mapping. Scientometrics. https://doi.org/10.1007/s1 11 192-009-0146-3

Venkataraman, S. (1997). The distinctive domain of entrepreneurship research. Advances in Entrepreneurship, Firm Emergence and Growth, 3, 119-138. https://doi.org/10.1108/S1074754020190000021009

Walley, E. E. L., Taylor, D. W., \& David. (2002). Opportunists, Champions, Mavericks...? Greener Management International, 38, 31-43.

Weaver, D. (2011). Can sustainable tourism survive climate change? Journal of Sustainable Tourism, 19(1), 5-15. https://doi.org/10.1080/09669582.2010.536242 
XU, X., \& Gursoy, D. (2015). A Conceptual Framework of Sustainable Hospitality Supply Chain Management. Journal of Hospitality Marketing and Management, 24(3), 229-259. https://doi.or $\mathrm{g} / 10.1080 / 19368623.2014 .909691$

Young, W., \& Tilley, F. (2006). Can Business Move Beyond Efficiency? The shift toward effectiveness and equity in the corporate sustainability debate. Business Strategy and the Environment, 15, 402-415. https://doi.org/10.1002/bse.510

Yu, L., Wang, G., \& Marcouiller, D. W. (2019). A scientometric review of pro-poor tourism research: Visualization and analysis. Tourism Management Perspectives, 30(January), 75-88. https://doi. org/10.1016/j.tmp.2019.02.005

Zupic, I., \& Čater, T. (2015). Bibliometric Methods in Management and Organization. Organizational Research Methods, 18(3), 429-472. https://doi.org/10.1177/1094428114562629

Contribuição de cada autor na construção do artigo

Pedro Chapaval Pimentel: Fundamentação teórica, coleta e análise dos resultados, redação e revisão.

Rivanda Meira Teixeira: Fundamentação teórica, estruturação, análise dos resultados e revisão. 